\title{
Characterisation of creep behaviour using the power law model in copper alloy
}

\author{
M.I.M. Ahmad ${ }^{1,2 *}$, J.L. Curiel Sosa ${ }^{1}$ and J.A. Rongong ${ }^{1}$ \\ ${ }^{1}$ Department of Mechanical Engineering, The University of Sheffield, Sir Frederick \\ Mappin Building, Mappin Street, S1 3JD Sheffield, United Kingdom \\ *Email: mimeorahmad1@ @ sheffield.ac.uk \\ ${ }^{2}$ Department of Mechanical \& Materials Engineering, \\ Faculty of Engineering and Build Environments, Universiti Kebangsaan Malaysia, \\ 43600 UKM Bangi, Selangor, Malaysia
}

\begin{abstract}
This paper presents a numerical strategy for the characterisation of the creep behaviour model of the copper alloy, which is widely used in aircraft applications under creep conditions. The high possibility of the material failing, while operating under load at an elevated temperature, has led to the important study of the creep lifetime prediction analysis, by presenting the Norton's rule based on the Power-law model to describe the secondary creep behaviour of the material. In order to demonstrate the nature of the creep formulation, the SOL 400 modules from MSC Nastran 2014 are implemented in order to conduct the uniaxial tensile test in $2000 \mathrm{~N}$ of applied load and $473 \mathrm{~K}$ of temperature condition. As a result, the exponential curve is formed from the relationship of the creep strain rate and stress, with a $5.1 \%$ error based on the value of the stress exponent, $n$, between the simulation and experimental results and this was still be acceptable because it was relatively small due to the formulation in the simulation. Consequently, a relation of the creep rate curve can then be plotted with respect to the load steps and the variation patterns due to the stress factor also being discussed. Therefore, the results show a good agreement, which indicates the capability of this model to give an accurate and precise estimation of the secondary creep behaviour of the materials.
\end{abstract}

Keywords: Characterisation; Norton's rule; secondary creep; creep strain rate curve.

\section{INTRODUCTION}

Since the 1950s, material engineers and engineering industry designers, particularly in the aircraft applications industry, have been concerned with the structural failure analysis in an attempt to improve flight safety. During the aircraft design process, many aspects need to be considered, and thus in order to assure aircraft safety, the material selection of the plane must be able to survive high stress and temperature environments for extended periods of time. When assessing the resistance of materials to deformation and failure under certain loads at high or low temperatures over long periods of time, particular attention must be given to a phenomenon known as creep. Creep is the permanent deformation and inelastic response of materials loaded with constant applied stress and a high temperature with a time function dependence $[1,2]$. The main cause of metal creep failure is nucleation, which is the growth and coalescence of cavities on the grain boundaries [3-5]. By modelling the behaviour of materials in a constitutive equation, the 
formation of the void, void growth, coalescence, micro-crack and crack propagation can be predicted mathematically.

In order to predict deformations and evaluate the lifetime of creep materials behaviour, many previous researchers used a numerical approach by implementing a finite element method (FEM) in their analyses. For example, a hyperbolic sine model is used as a constitutive model for the high temperature creep of ASTM A992 steel, in which this is fitted to the experimental creep data [1]. The isothermal creep method is established in order to describe the high-temperature creep behaviour of the SA-508 metal material and serves as a reference for the safety assessment of the components of reactor pressure vessels [6]. Then, the creep behaviour of the 2024-T3 aluminium alloy at high temperatures $\left(150-200{ }^{\circ} \mathrm{C}\right)$ has been described numerically by implementing the Power Law model [7]. Moreover, to validate the results from the computational approach, the experimental creep test is being prepared as a benchmark. Austenitic stainless steels (316LN) with different batches are subjected to numerous creep tests at various stresses and temperatures between $525^{\circ} \mathrm{C}$ and $750{ }^{\circ} \mathrm{C}$ up to nearly 50000 hours [8]. As a result, the extrapolation of the stress-lifetime curves obtained at high stress leads to a massive overestimation of lifetimes at low stress and the model proposed by Riedel is implemented for comparison purposes. Hence, much research effort needs to be invested now in developing appropriate constitutive equations to solve the numerical shortcomings of the creep problem [9]. In this study, a copper alloy, which is widely used in the fabrication of aircraft structure components, is investigated to characterise the secondary (or steady state) creep behaviour using Norton's rule, based on the Power Law model towards the formation of the material creep behaviour. The capability of this model becomes a driving force to develop the numerical analysis of the creep problem, and the experimental data obtained in order to validate the results.

\section{METHODS AND MATERIALS}

\section{Creep Experiment}

A uniaxial creep test was performed in accordance with ASTM D 2990, with a constant external force and temperature condition. The material properties and the dimensions of the specimen for the creep test are shown in Table 1 [10].

Table 1. Material properties and dimensions.

\begin{tabular}{cc}
\hline Young Modulus, $\mathrm{E}$ & $120 \mathrm{GPa}$ \\
Poison Ratio, $v$ & 0.36 \\
Load force, $\mathrm{P}$ & $2000 \mathrm{~N}$ \\
Temperature, $\mathrm{T}$ & $473 \mathrm{~K}$ \\
Dimensions & $150 \mathrm{~mm} \times 25 \mathrm{~mm} \times 10 \mathrm{~mm}$ \\
\hline
\end{tabular}

\section{Simulation Analysis}

Computational work was conducted using the MSC Nastran 2014 software to study the characterisation of secondary creep behaviour in the tensile creep test simulation. This software offers SOL 400 modules, which have the nonlinear capabilities to analyse a wide variety of structural problems subjected to geometric and material nonlinearity. In order to model the creep characteristics, a few options need to be considered in the analysis such as the CREEP option to identify the creep time behaviour of the material, the MATVP option to describe the materials properties and the NLSTEP bulk data entry for 
controlling the time integration in the finite element program. The flow diagram of the non-linear problem analysis for the simulation work is shown in Figure 1. This analysis is a quasi-static analysis, using adaptive time stepping for real-time creep, with geometric and material nonlinearity due to large strain and creep. The uniaxial tensile creep test is illustrated computationally in the 2D-analysis and the discretisation is shown in Figure 2. This model consists of 36, 4-node plane strain elements and 49 nodes.

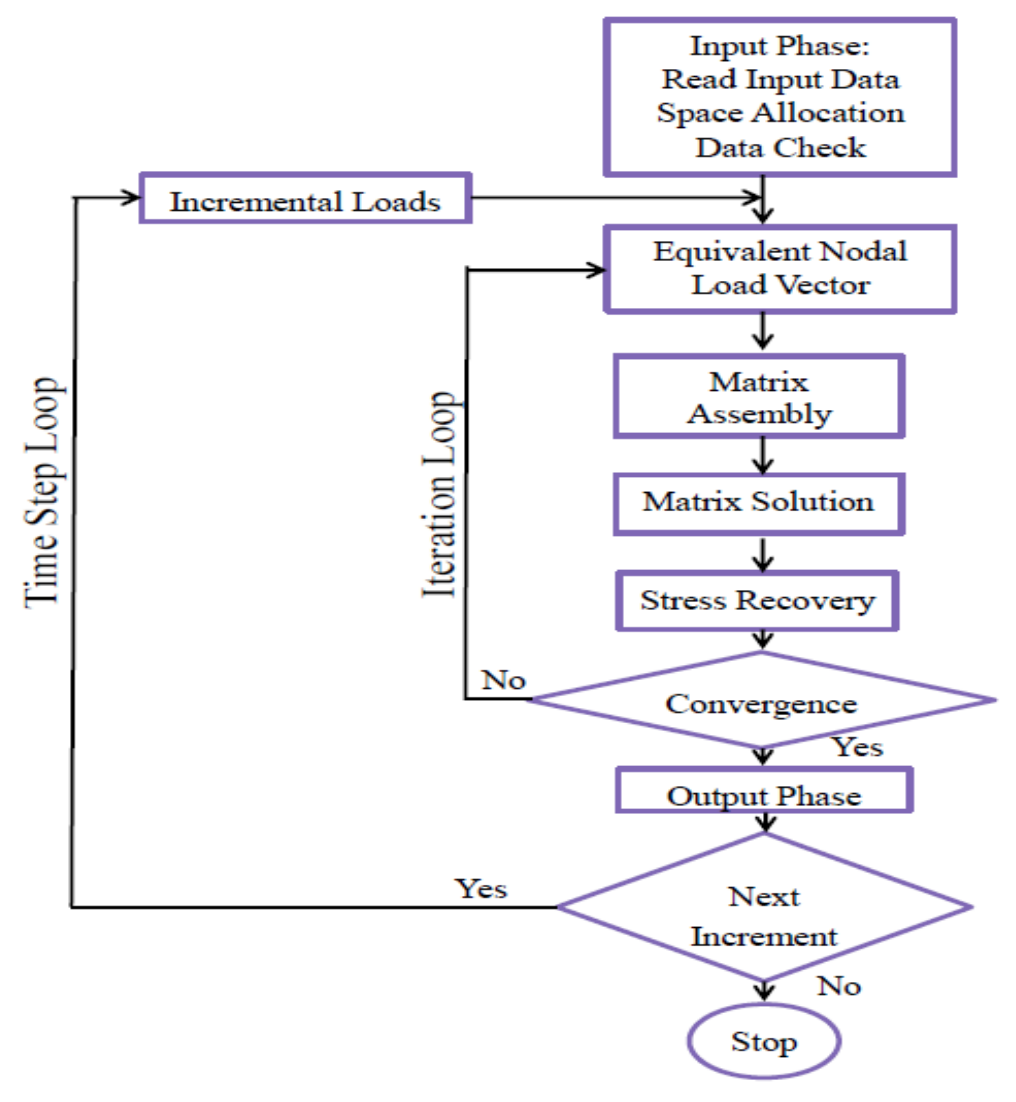

Figure 1. Flow diagram of the non-linear creep analysis.

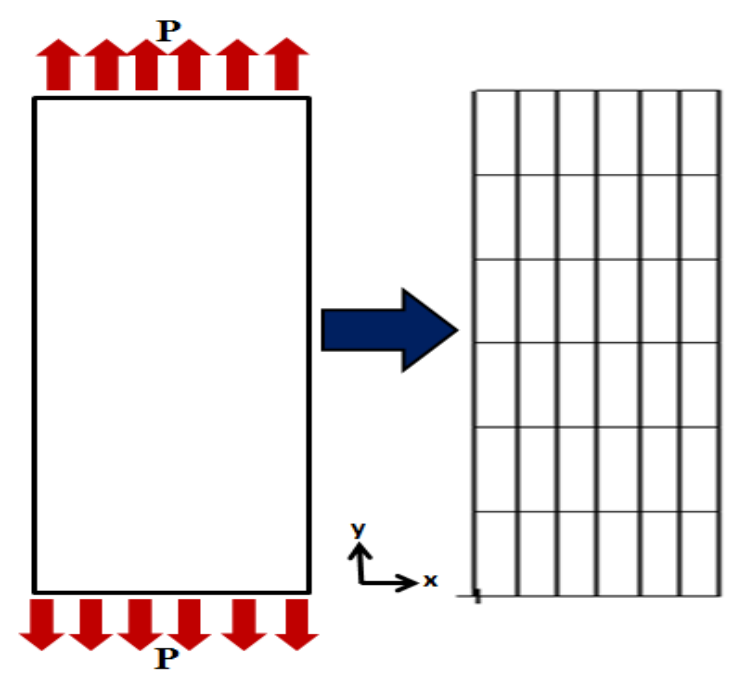

Figure 2. Sketch of the specimen geometry with a 2D-discretisation of the plane strain. A boundary condition was fixed at the $z$-axis $(z$-axis $=0)$. 


\section{MATHEMATICAL MODELLING}

Conventional creep behaviour is based on a Von Mises creep potential with an isotropic behaviour described by the equivalent creep law:

$$
\dot{\varepsilon}^{c}=f\left(\sigma, \varepsilon^{c}, T, t\right)
$$

The material behaviour is therefore described by:

$$
\Delta \varepsilon^{c}=\dot{\varepsilon}^{c} \frac{\partial \bar{\sigma}}{\partial \sigma^{\prime}} \Delta t
$$

where $\frac{\partial \bar{\sigma}}{\partial \sigma^{\prime}}$ is the outward normal to the current Von Mises stress surface and $\dot{\varepsilon}^{c}$ is the equivalent creep strain rate. There are two numerical procedures used in implementing creep behaviour. The default is an explicit procedure in which the above relationship is implemented in the program by an initial strain technique. In addition, a pseudo-load vector due to the creep strain increment is added in the stiffness equation as:

$$
K \Delta u=\Delta P+\int_{V} \beta^{T} D \Delta \varepsilon^{c} d v
$$

where $\mathrm{K}$ is the stiffness matrix, $\Delta u$ is the incremental displacement, $\Delta P$ represents the incremental nodal force vector and $\int_{V} \beta^{T} D \Delta \varepsilon^{c} d v$ is the pseudo-load vector due to the creep strain increment in which $\beta$ is the strain displacement relation and D is the stressstrain relation [11].

The dependence of the equivalent creep strain rate on any independent parameter can be given directly in the power law form. The equivalent creep strain rate is:

$$
\dot{\varepsilon}^{c}=A \sigma^{n}\left(\varepsilon^{c}\right)^{m} T^{p}\left(q t^{q-1}\right)
$$

where $\mathrm{A}, \mathrm{p}$ and $\mathrm{q}$ are the material constants, $\mathrm{n}$ is a stress exponent, and $\sigma, \varepsilon^{c}, T$ and $t$ are the equivalent stress, equivalent creep strain, temperature and time, respectively. For this analysis, only the stress and temperature parameters will be identified. Equation 5 is simplified as:

$$
\dot{\varepsilon}^{c}=A \sigma^{n} T^{p}
$$

The expression, $\dot{\varepsilon}_{c} \propto \sigma^{n}$, is sometimes called Norton's law, and provides the basic power law relationships, which have been widely used to describe high-temperature creep behaviour. By referring to the experimental data [10], all parameters are determined, in which the value of $A=6.3167 \mathrm{E}-14, n=5.6546$ and $p=0.3$.

\section{RESULTS AND DISCUSSION}

\section{Mesh Convergence}

In order to identify the adequate size of meshing, a mesh convergence curve was created through the analysis. Figure 3 shows the mesh convergence curve that exhibits the best relationship between the stress and the number of elements of the specimen structure in the finite element analysis. The stress increases with an increasing number of elements, 
until up to 30 elements; it starts to converge and reaches a stable form. Therefore, 36 elements were chosen in this analysis to achieve mesh convergence stability and to obtain a symmetrical geometry number of elements.

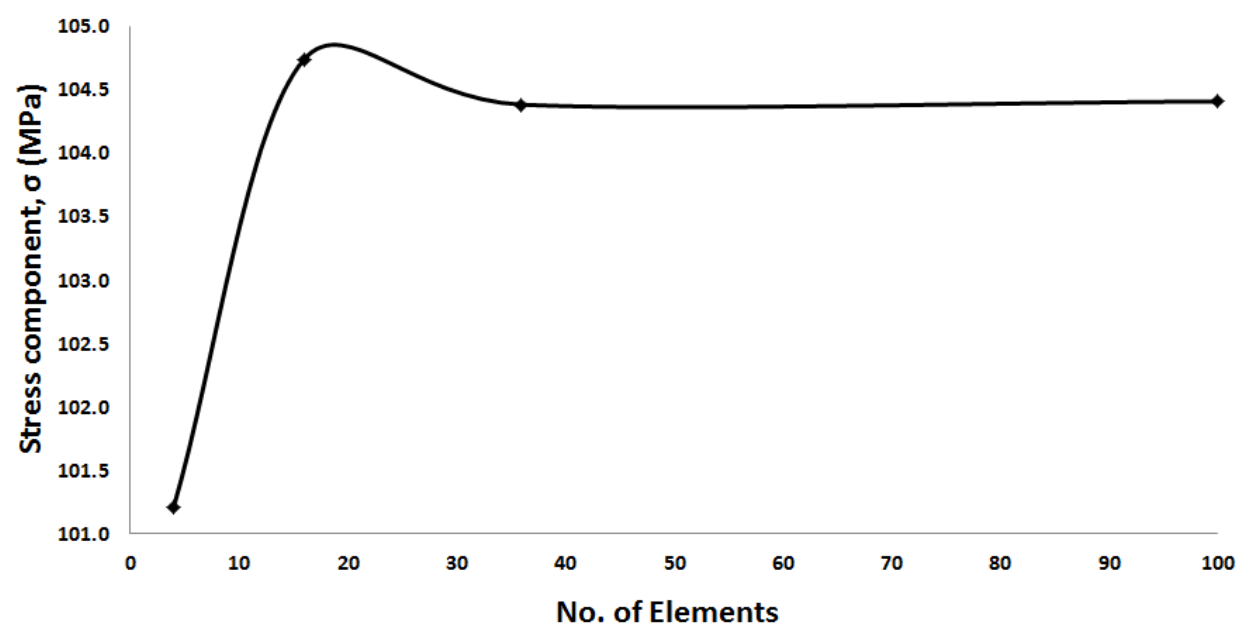

Figure 3. Mesh convergence curve.

\section{Power Law of Creep}

Firstly, the relationship of the creep strain rate and stress was analysed as shown in Figure 4. From the graph, the exponential curve is formed, which actually expresses a Norton's Law relation. The creep strain rate of the copper alloy increased with the increasing of stress respectively [12]. Theoretically, the function of the Power Law creep depends on the variable of the material constant, $\mathrm{A}$, and the stress exponent, $\mathrm{n}$, of the material [13]. An experimental result was also plotted in the same graph to clarify the accuracy of the simulation's results. The graph clearly shows a similar pattern of exponential curves between the simulation and experimental results. The value of the stress exponent, $n$, between both methods was compared to identify the precision of the result, thus bringing the $5.1 \%$ error, and this was still acceptable because it was relatively small due to the formulation in the simulation.

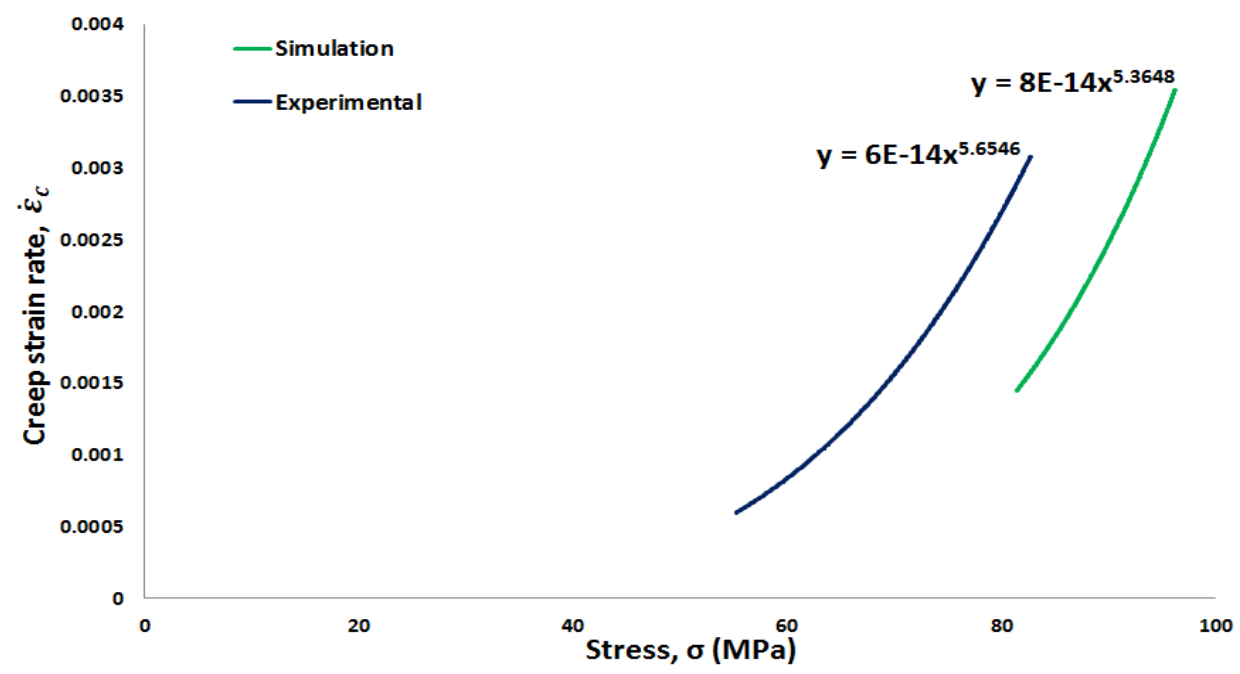

Figure 4. Power Law relation between the creep strain rate and stress for the experimental and simulation results. 


\section{Stress Dependence Analysis}

Secondly, in order to determine the secondary creep behaviour of the copper alloy, the creep rate curve relationship between the creep strain and creep strain rate with respect to time (load steps) was plotted (as shown in Figures 5 and 6). Figure 5 shows the schematic creep curves at the same temperature that represents the secondary creep strain with load steps. In order to see the influence of the stress in the creep strain formation, the values of stress were manipulated through the analysis and the results obtained are plotted together. By increasing the stress respectively, the secondary creep strain increases and the rupture lifetime of the material decreases on increment of the stress. The pattern of the plotted creep curves shows similar results to those studied by [1,7, 14-17], which imitate the secondary creep regime. Moreover, the creep strain rate forms an approximately steady state as shown in Figure 6. The stresses applied typically affect the formation of the creep strain rate and also the rupture lifetime of the material.

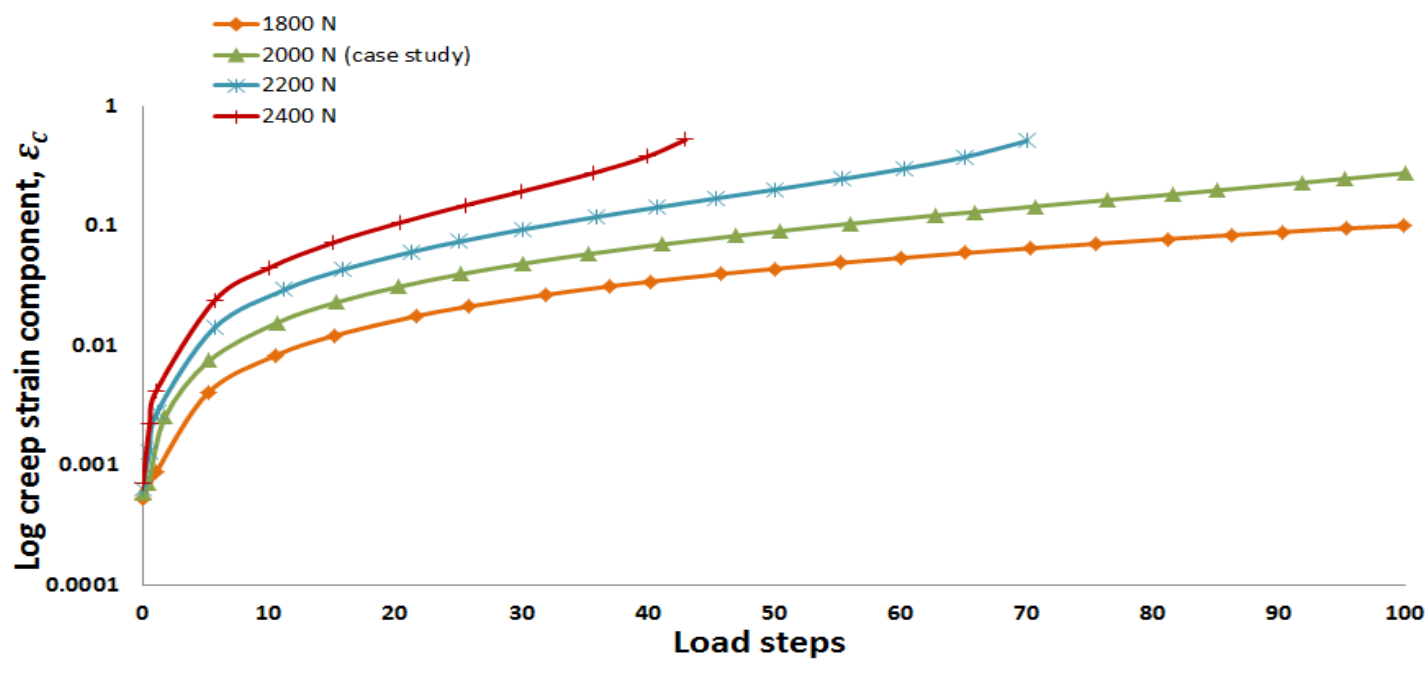

Figure 5. Creep strain as a function of the time/load steps at a temperature of $473 \mathrm{~K}$.

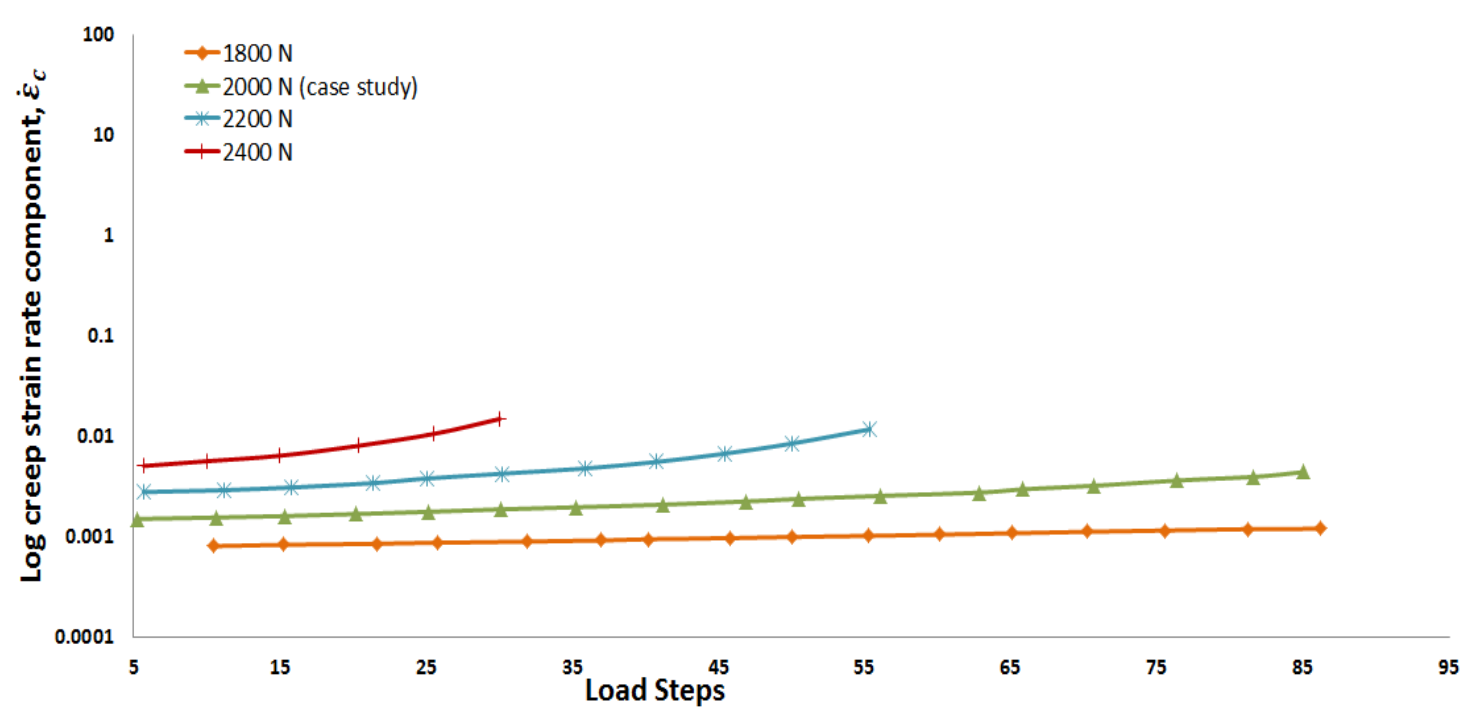

Figure 6. Creep strain rate as a function of the time/load steps at a temperature of $473 \mathrm{~K}$. 


\section{CONCLUSIONS}

This study is to characterise the secondary creep behaviour of the copper alloy using the Power-law as a constitutive creep model. To develop this case study, a computational approach was presented in the uniaxial creep test with constant stresses and temperature condition. As a result, the graph of the creep strain rate against stress was identified as being representative of the secondary creep behaviour, and with $5.1 \%$ of error, there was good agreement between the simulation model and the experimental results. Moreover, the graphs for the creep strain and the creep strain rate with load steps were presented, which represent the secondary creep regime. The variations of the stresses give the primary factor that affects the formation of the creep strain and also the rupture life of the material. The highest value of stresses creates a significant impact toward the creep strain and shortens the failure lifetime of the material. Therefore, the simulation model, based on a Norton's Law equation, provides the basics for the Power Law relationships that have been widely used to describe secondary creep behaviour. In order to develop the knowledge of creep failure, the estimation creep model will be drawn up to predict the creep damage behaviour by considering the creep crack initiations and the creep crack growth model of materials.

\section{ACKNOWLEDGEMENTS}

The authors would like to express their gratitude to the University of Sheffield, Universiti Kebangsaan Malaysia and Ministry of Education Malaysia through the fund of LSF and SLAB-SLAI (KPM-UKM) for supporting this research project.

\section{REFERENCES}

[1] Cowan M, Khandelwal K. Modeling of high temperature creep in ASTM A992 structural steels. Engineering Structures. 2014;80:426-34.

[2] Jing-Tian L, Xi-Ming R, Jian-Lu W, Bang-Qiang Z, Xi-Jing N. A universal function of creep rate. Chinese Physics B. 2015;24:093401.

[3] Yao H-T, Xuan F-Z, Wang Z, Tu S-T. A review of creep analysis and design under multi-axial stress states. Nuclear Engineering and Design. 2007;237:196986.

[4] Saad AA, Bachok Z, Sun W. A study on the damage evolution of P91 steel under cyclic loading at high temperature. International Journal of Automotive and Mechanical Engineering. 2016;13:3564-73.

[5] Daud R, Ariffin AK, Abdullah S. Validation of crack interaction limit model for parallel edge cracks using two-dimensional finite element analysis. International Journal of Automotive and Mechanical Engineering. 2013;7:993-1004.

[6] Xie L-J, Ren X, Shen M-X, Tu L-Q. Parameter correlation of high-temperature creep constitutive equation for RPV metallic materials. Journal of Nuclear Materials. 2015;465:196-203.

[7] Maximov J, Duncheva G, Anchev A, Ichkova M. Modeling of strain hardening and creep behaviour of 2024T3 aluminium alloy at room and high temperatures. Computational Materials Science. 2014;83:381-93.

[8] Cui Y, Sauzay M, Caes C, Bonnaillie P, Arnal B. Modeling and experimental study of long term creep damage in austenitic stainless steels. Procedia Materials Science. 2014;3:122-8. 
[9] Mackerle J. Creep and creep fracture/damage finite element modelling of engineering materials and structures: an addendum. International journal of pressure vessels and piping. 2004;81:381-92.

[10] Evans RW, Wilshire B. Introduction to creep. The Institute of Materials(UK), 1993. 1993:115.

[11] Nastran M. User's Guide for SOL. 2015. p. 558 - 63.

[12] Larsson J. Evaluation of current methods for creep analysis and impression creep testing of power plant steels: KTH Royal Institute of Technology; 2012.

[13] Takagi H, Dao M, Fujiwara M. Prediction of the constitutive equation for uniaxial creep of a power-law material through instrumented microindentation testing and modeling. Materials Transactions. 2014;55:275-84.

[14] Zhang Z, Cui P, Lv Z, Liu N, Zhu S-P, Huang H-Z. Notice of Retraction Creep life prediction model of aircraft turbine disc alloy based on continuum damage mechanics. Quality, Reliability, Risk, Maintenance, and Safety Engineering (QR2MSE), 2013 International Conference on: IEEE; 2013. p. 1018-21.

[15] Li L-T, Lin Y, Zhou H-M, Jiang Y-Q. Modeling the high-temperature creep behaviors of 7075 and 2124 aluminum alloys by continuum damage mechanics model. Computational Materials Science. 2013;73:72-8.

[16] Lin Y, Xia Y-C, Chen M-S, Jiang Y-Q, Li L-T. Modeling the creep behavior of 2024-T3 Al alloy. Computational Materials Science. 2013;67:243-8.

[17] Geng L, Gong J, Liu D, Jiang Y. Damage analysis and life prediction of a main steam pipeline at elevated temperature based on creep damage mechanics. Sustainable Power Generation and Supply, 2009 SUPERGEN'09 International Conference on: IEEE; 2009. p. 1-6. 